\title{
Self-organized defect strings in two-dimensional crystals
}

\author{
Wolfgang Lechner, ${ }^{1,2}$ David Polster, ${ }^{3}$ Georg Maret, ${ }^{3}$ Peter Keim, ${ }^{3}$ and Christoph Dellago ${ }^{4}$ \\ ${ }^{1}$ Institute for Quantum Optics and Quantum Information, Austrian Academy of Sciences, 6020 Innsbruck, Austria \\ ${ }^{2}$ Institute for Theoretical Physics, University of Innsbruck, 6020 Innsbruck, Austria \\ ${ }^{3}$ Department of Physics, University of Konstanz, D-78457 Konstanz, Germany \\ ${ }^{4}$ Faculty of Physics, University of Vienna, Boltzmanngasse 5, 1090 Vienna, Austria
}

(Received 15 July 2013; published 10 December 2013)

\begin{abstract}
Using experiments with single-particle resolution and computer simulations we study the collective behavior of multiple vacancies injected into two-dimensional crystals. We find that the defects assemble into linear strings, terminated by dislocations with antiparallel Burgers vectors. We show that these defect strings propagate through the crystal in a succession of rapid one-dimensional gliding and rare rotations. While the rotation rate decreases exponentially with the number of defects in the string, the diffusion constant is constant for large strings. By monitoring the separation of the dislocations at the end points, we measure their effective interactions with high precision beyond their spontaneous formation and annihilation, and we explain the double-well form of the dislocation interaction in terms of continuum elasticity theory.
\end{abstract}

DOI: 10.1103/PhysRevE.88.060402

PACS number(s): 61.72.-y, 82.70.Dd

The plasticity and mechanical strength of solids is essentially governed by the dynamics of dislocations, topological defects generated during plastic deformation [1]. Recent research has focused on the properties and effects of such defects in novel two-dimensional nanomaterials. In graphene, for instance, dislocations generated, say, by irradiation can significantly alter the electronic and mechanical properties of the material, and the ability to control them will be key for technological application [2-4]. The interaction between antiparallel dislocations also plays a central role in twodimensional melting, which, according to the KosterlitzThouless-Halperin-Nelson-Young theory [5], is mediated by the formation and subsequent dissociation of dislocation pairs leading to the loss of quasi-long-range translational order [6-9]. Due to the remarkable experimental advances of recent years, such as the development of optical tweezers and confocal microscopy [6,10-15], the direct observation of the structure and dynamics of defects is now possible with single-particle resolution. However, measuring the interaction of dislocations experimentally for a wide range of distances and angles is challenging, mainly due to the lack of a systematic way to generate and control dislocations beyond their spontaneous formation and annihilation [6].

In this Rapid Communication, we study the dynamics of self-organized defect strings and exploit their properties to measure the interaction of dislocations using a planar crystal of colloidal particles with tuneable interactions as the model system. We confirm experimentally, as shown in Fig. 1, that multiple vacancies introduced artificially into the system rapidly cluster, driven by the attractive defect interaction [16-18]. Furthermore, we confirm, that the vacancy clusters arrange into linear strings which cannot be understood from linear elasticity theory [18]. These defect strings are terminated by two dislocations of antiparallel Burgers vectors and can be considered the two-dimensional analogs of the prismatic dislocation loops [19-22] forming in irradiated three-dimensional materials. In contrast to dislocation pairs forming spontaneously by thermal excitation, the dislocations resulting from defect clustering do not annihilate due to the geometrical constraints imposed by the defect string. While the separation of the dislocations perpendicular to the Burgers vectors is fixed and depends on the number of vacancies in the string, the dislocations are mobile in the parallel direction. As discussed below, we use the statistics of this motion, collected for various string lengths, to reconstruct the complete interaction Hamiltonian of dislocation pairs with antiparallel Burgers vectors. In particular, we find an interaction of double-well form, as predicted by elasticity theory.

Our system consists of a monolayer of superparamagnetic colloidal particles confined by gravity to a flat water-air interface $[23,24]$. A magnetic field of strength $H$ perpendicular to the interface induces magnetic dipole moments of magnitude $\chi H$, leading to the dipolar pair interaction

$$
\beta v(r)=\frac{3^{3 / 4} \Gamma}{(2 \pi)^{3 / 2}}\left(\frac{a}{r}\right)^{3} .
$$

Here, $\beta=1 / k_{B} T$ with the Boltzmann constant $k_{B}$ and temperature $T, r$ is the particle-particle distance, $a$ is the lattice spacing of the perfect crystal and $\Gamma=\beta\left(\mu_{0} / 4 \pi\right)(\chi H)^{2}(\pi \rho)^{3 / 2}$, where $\mu_{0}$ is the permeability of vacuum and $\rho$ is the area density of the crystal. The dimensionless parameter $\Gamma$, which acts as an inverse temperature and can be tuned by varying the magnetic field, completely determines the structural properties of the system. Vacancies are generated by a fiber-coupled optical tweezer, where the objective can be moved in $x, y$, and $z$ directions for distances larger than the field of view. The colloids are trapped in the laser focus for moderate field strength, but for high field strength $(500 \mathrm{~mW}, 100 \times$ tweezer objective, NA $0.73, \mathrm{Ar}^{+}$laser) light pressure is strong enough to push particles through the interface, removing them from the monolayer. We also study the vacancy strings using $N V T$ Monte Carlo (MC) simulations with local trial moves.

The sequence in Figs. 1(a)-1(f) illustrates the formation of a defect string with $N=9$ vacancies in the experiment. Dislocations are identified as pairs of five and seven coordinated particles identified with Voronoi construction. Even though there are nine vacancies present in the system, only two dislocations remain in the equilibrated string configuration [see Fig. 1(f)]. The region between the two dislocations is a prefect crystal, in which each particle has exactly six 

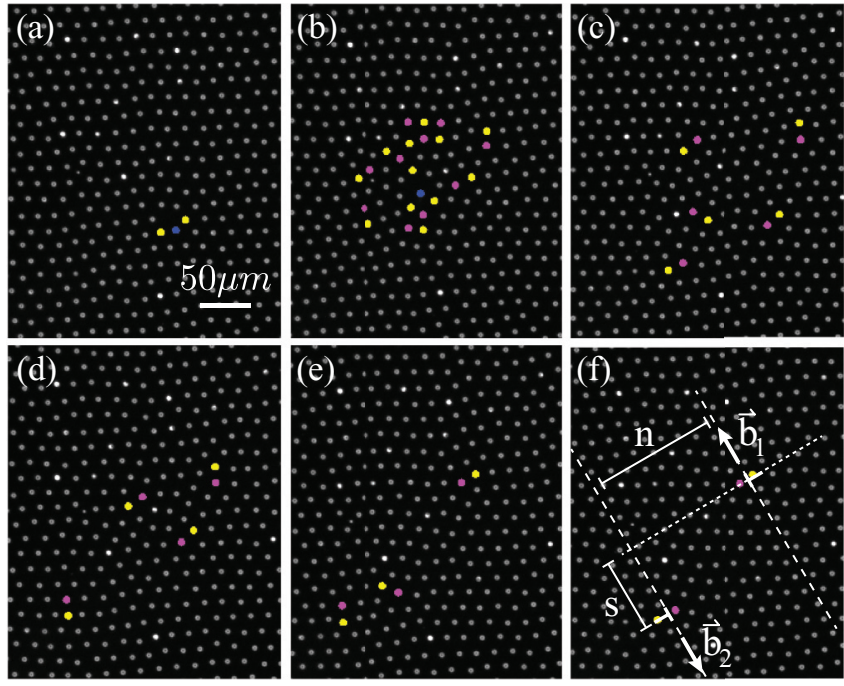

FIG. 1. (Color online) Formation of a defect string with $N=9$ vacancies observed in our experiments. (a)-(b) The initial configuration is created by removing 9 particles with strong laser pulses from a perfect triangular colloidal crystal. Along the sequence (c)-(f), the vacancies gradually coalesce and annihilate, eventually leaving behind a pair of dislocations with Burgers vectors of opposite direction. Colors distinguish particles with 8 neighbors (blue), 7 neighbors (pink), 6 neighbors (gray), and 5 neighbors (yellow). After about $6 \mathrm{~min}$ the vacancies have clustered into a vacancy string terminated by two dislocations. This behavior is in perfect agreement with Monte Carlo simulations performed at the conditions of the experiments. (f) To follow the dynamics of the string in detail we monitor the position of the two dislocations, each of which corresponds to a 5-7 coordinated particle pair and is indicated by a white T symbol. The dislocations diffuse along parallel lattice lines, indicated by dashed lines, parallel to the Burgers vectors $\mathbf{b}_{1}$ and $\mathbf{b}_{2}$. While the perpendicular separation $n$ between the dislocations is fixed by the number $N$ of vacancies introduced into the system, the parallel distance $s$ changes as the dislocations move under the influence of their effective interaction.

neighbors. This remarkable formation of linear strings rather than defect clusters can be understood from the complex interactions of vacancies, which cannot be written in terms of pair interaction $[17,18]$. We note that through comparison with the underlying ideal lattice, one can locate the entire string of vacancies that connects the dislocations at the string endpoints [18].

Analyzing trajectories obtained from our video microscopy experiments and computer simulations in terms of defect positions, we find that defect strings propagate through the system in a sequence of fast gliding motions and rare rotations (see Fig. 2). During the gliding phase, the vacancies and dislocations at the string endpoints move in a direction that is parallel to the Burgers vectors of the dislocations and coincides with one of the three symmetry axes of the crystal. In the direction perpendicular to the direction of motion, distances between defects remain constant. As a consequence, the string endpoints move on parallel lines, separated by $N$ lattice rows. In this gliding phase, the motion of the defect string can be viewed as the diffusion of the two endpoint dislocations along parallel lines under the influence of their

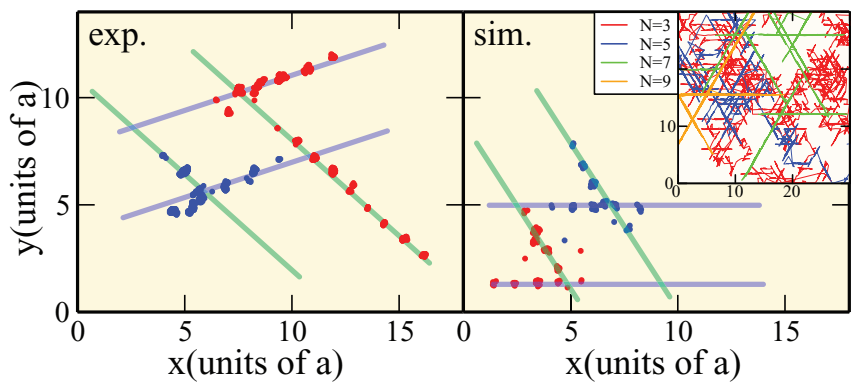

FIG. 2. (Color online) Dynamics of defect strings. Left: Trajectory obtained from the experiment of the dislocation positions, shown as red and blue dots. Dislocations first move along two parallel lines in one of the main lattice directions and then change their direction of motion during a rapid reorientation event that turns the Burgers vectors of the dislocations by $\pi / 3$. This combination of gliding and rotation is consistent with computer simulations (right). As discussed below, rotation events can only occur at particular points [see Fig. 4 (top right panel)]. The long-time diffusion of defect strings of various lengths from $N=3$ to $N=9$ is depicted as an inset. Here, each point corresponds to the center of mass of the two dislocations indicating the position of the string. While the diffusion constant is roughly independent of $N$, the rotation probability decreases exponentially with $N$ leading to long gliding periods for large $N$ (green).

mutual effective interaction. In the direction of motion the dislocations interact attractively at large distances, such that the two dislocations can diffuse only as a coupled pair. On short time scales, the dislocations thus diffuse freely with diffusion constant $D_{\text {dis }}$, while at long time scales the diffusion of the dislocations is governed by the diffusion constant $D_{\text {string }}=$ $D_{\text {dis }} / 2$ [26]. This allows one to extract the diffusion constant of individual dislocations from the diffusion of the string. Diffusion constants obtained from computer simulations and experiments, depicted in Fig. 3, agree within the statistical accuracy of the results. Remarkably, the diffusion constant is independent of the string length for $N>5$, corroborating the view of the string motion as the diffusion of two coupled dislocations. Thus, the number of vacancies in the string

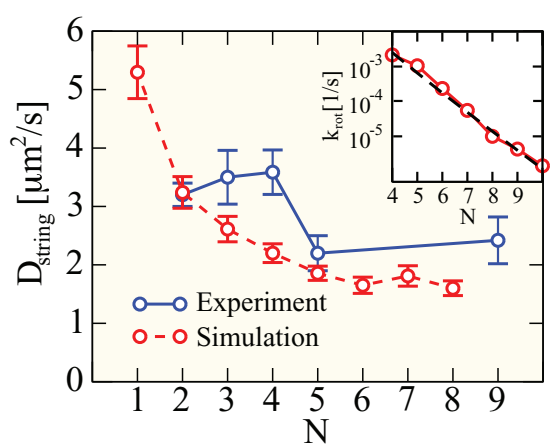

FIG. 3. (Color online) Diffusion constant $D_{\text {string }}$ of vacancy strings as a function of defect number $N$. Experimental results (blue solid line) are shown together with results of the simulations (red dashed line). In the simulations, averages and error bars are calculated from 12 blocks of $4 \times 10^{6} \mathrm{MC}$ sweeps each. The inset shows the rotation rate $k_{\text {rot }}$ [25] of vacancy strings as a function of $N$. A fit of $k(N) \propto \exp \left(-\beta N e_{a}\right) / N$, shown as a dashed line, yields an activation energy per defect of $e_{a}=1.13 k_{\mathrm{B}} T$. 
influences the time at which the long-time diffusion regime is reached, but not the diffusion constant itself [26].

The phases of facile gliding motion are interrupted by rotation events that change the direction of motion of the dislocations, allowing the diffusion to become two dimensional. Rotations can take place only at specific rotation points at the intersections of the lattice lines indicated by dashed lines in Fig. 4 (top right), along which the endpoint dislocations have constant perpendicular distance. During such a rotation event, which can be detected based on the directions of the Burgers vectors or the sequence of dislocation positions, the string changes from one of the three possible directions of motion to another. Examples of two rotation events observed in the experiments and computer simulations are shown in Figs. 2(a) and 2(b) for experiment and simulation, respectively. Since rotation events are rare, we could determine the rotation rate reliably only for the long trajectories obtained from our computer simulations [Fig. 2(b) inset]. The resulting patterns of diffusion can be understood from the rotation rate and the diffusion constant. As shown in the inset of
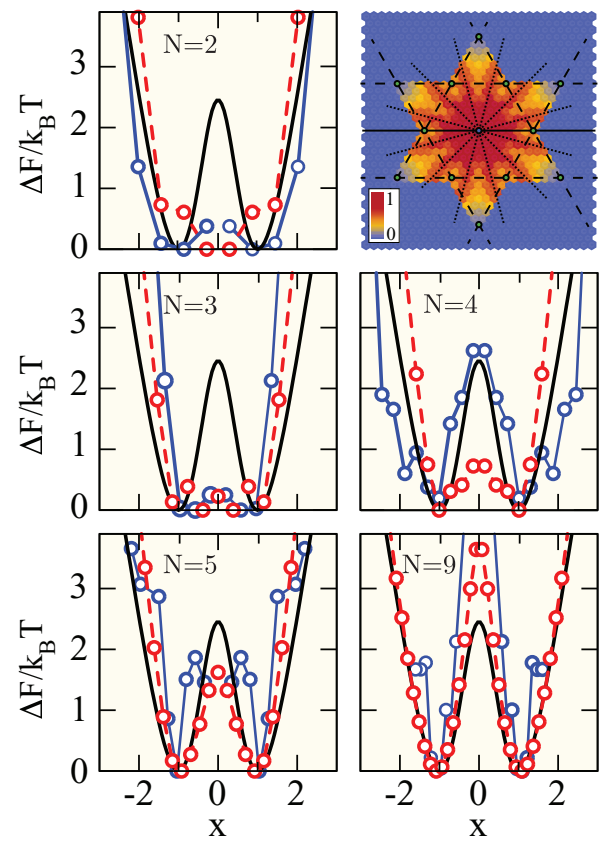

FIG. 4. (Color online) Free energy $\beta \Delta F$ as a function of the scaled distance $x=2 s /(\sqrt{3} N)$ in the direction of motion for defect strings with lengths from $N=2,3,4,5$, and 9. Experimental results (blue solid lines) are shown together with results of simulations (red dashed lines) and the prediction of elasticity theory (black line) [see Eq. (2)]. The top right inset depicts a color coded histogram of vacancy locations for $N=8$ and one string end at the origin (blue dot). The vacancy string follows the same double-well pattern with angle $\pi / 4$ (dotted lines) as the endpoints. The relative distance between dislocations (dashed lines) is constrained to six lines with 12 rotation points (green dots) where the string can change the gliding orientation. These rotation points are the only points where the constraint of perpendicular distance $n$ is satisfied for two different orientations. Results from computer simulations are obtained using $N=3120$ under periodic boundary conditions. Simulations carried out with different system sizes indicate that finite-size effects can be neglected.
Fig. 3, the rotation rate $k_{\text {rot }}$, defined as the number of rotation events per second, decreases rapidly with string length. The particular functional form of $k_{\text {rot }}(N)$ can be understood as follows. The probability to observe a rotation at a given time is the product of the probability to be at a rotation point multiplied by the probability to change the direction of motion at this point. The probability to be at a rotation point decreases as $1 / N$ simply because of the number of locations available to the string endpoints is proportional to $N$. At a rotation point, a string can change its direction by a collective slip of two rows of $N$ particles past each other in the region between the endpoint dislocations. This collective rearrangement involves an activation energy that increases linearly with the string length, $E_{a}=N e_{a}$. Including both contributions to the rotation probability, one expects a rotation rate $k_{\text {rot }}(N) \propto \exp \left(-\beta N e_{a}\right) / N$. A fit of this expression to the simulation results yields good agreement, as shown in the inset of Fig. 3.

To explore the effective interaction between the two dislocations located at the endpoints of the strings, we have determined the probability $P(s)$ of finding the dislocations at distance $s$ in the direction of motion [see Fig. 1(d)]. The probabilities $P(s)$ are obtained by histogramming the distances $s$ along trajectories recorded in the experiments and in the simulations. We ignore configurations in which additional dislocation pairs arise through thermal fluctuations (10-20\% of the configurations) and assume that the spontaneous formation of additional dislocations does not influence the defect string configuration. Therefore, neglecting these multidislocation configurations does not affect the calculated effective interactions given by $F(s)=-k_{\mathrm{B}} T \ln P(s)$. In Fig. 4, effective interactions $\beta F$, displayed as a function of the scaled coordinate $x=2 s /(\sqrt{3} N)$, are compared to the predictions of elasticity theory for various string lengths $N$. The effective interactions obtained from our experiments agree well with the results of the computer simulations with deviations that are most likely due to the limited statistics of the experimental results.

For strings longer than $N>3$, both experiments and simulations yield effective interactions of double-well form with minima at $x= \pm 1$ corresponding to $s= \pm \sqrt{3} N / 2$, and a central barrier at $x=0$. As a consequence, for all string lengths the defect strings are preferentially oriented at an angle of $\pi / 4$ with respect to the high symmetry directions, and individual defects follow this $\pi / 4$ pattern (see Fig. 4 top right). The double-well form of the dislocation interaction and, in particular, the surprising defect string alignment, differing from the high symmetry directions of the hexagonal lattice, can be rationalized in terms of continuum elasticity theory. In two dimensions, two dislocations with Burgers vectors $\mathbf{b}_{1}$ and $\mathbf{b}_{2}$ and separated by $\mathbf{R}$ are predicted to interact with energy $\beta F=-(K / 4 \pi)\left[\left(\mathbf{b}_{1} \cdot \mathbf{b}_{2}\right) \ln R-\left(\mathbf{b}_{1} \cdot \mathbf{R}\right)\left(\mathbf{b}_{2} \cdot \mathbf{R}\right) / R^{2}\right]$ [27], where $K$ is Young's modulus [28,29]. For a string of $N$ vacancies, the geometry of the dislocations can be described by $\mathbf{b}_{1}=(-1,0), \mathbf{b}_{2}=(1,0)$, and $\mathbf{R}=(s, N \sqrt{3} / 2)$, yielding the effective interaction

$$
\beta F(x)=\frac{K}{8 \pi}\left(\frac{1-x^{2}}{1+x^{2}}+\ln \frac{1+x^{2}}{2}\right) .
$$


Note that this expression is valid for an isotropic system irrespective of the symmetry of the underlying crystal lattice to which no reference is made. The free energy of Eq. (2) is a symmetric double well with minima at $x= \pm 1$ separated by a barrier of height $h=\beta F(0)=(K / 8 \pi)(1-\ln 2)$ located at $x=0$. Thus, the barrier height depends on Young's modulus $K$ but not on the string length $N$. As can be seen in Fig. 4, for sufficiently long defect strings the free energy obtained from elasticity theory reproduces both the experimental and numerical results to a remarkable extent. In particular, the positions of the minima, which are related to the preferential $\pi / 4$ orientation of the strings, are predicted very accurately by elasticity theory for $N>3$. For short strings, elasticity theory fails at short distances since the discrete lattice becomes noticeable and for $N=2$ the potential becomes a single well. While the general shape of the free energy and the position of the minima are predicted well by elasticity theory, the predicted height of the barrier at $x=0$ differs significantly from the simulation results. Barrier heights obtained in our simulations seems to converge to a constant value for growing string length as expected from elasticity theory. This height, however, exceeds the prediction of linear elasticity by almost $50 \%$, possibly due to nonlinear elastic interactions.

In summary, we have used a combination of experiment and computer simulations to characterize the motion and interactions of defects in two-dimensional crystals with singleparticle resolution. We have found that vacancies introduced artificially into the system coalesce into string-like clusters that are almost as mobile as single vacancies. The dynamics of these strings consists of long stretches of fast diffusive gliding in one dimension punctuated by rotations at specific rotation points. For rotations, a collective rearrangement that reorients the dislocations terminating the vacancy string is needed. From the statistics of dislocation positions tracked with video microscopy we reconstruct the effective interactions between dislocations in two dimensions and the double well predicted by continuum elasticity. Based on our computer simulations, we predict that analogous clustering phenomena occur for interstitials introduced into two-dimensional crystals, providing another way to study defect clustering and dislocation interactions in two-dimensional materials. In future work we will study defect clustering and interactions in binary systems as well as in monolayers on a substrate and in systems with anisotropic interactions, which can be realized in our model system by tilting the magnetic field controlling the interactions between particles. In particular, it will be interesting to study the influence of such defects on the mechanical properties of the system. Approaches analogous to the ones used here to explore defects in colloidal crystals could be applied to directly probe interactions of defects generated by radiation damage in two-dimensional nanomaterials such as graphene or boron nitride sheets in high-resolution transmission electron microscopy experiments [3].

This research was supported by the Austrian Science Fund (FWF) within the SFB ViCoM (Projekt F41) and P 24681-N20. Simulations were carried out on the Vienna Scientific Cluster (VSC). P.K. and G.M. acknowledge financial support for the experimental part from the German Research Foundation, SFB-TR6 Project C4.
[1] G. I. Taylor, Proc. R. Soc. A 145, 362 (1934).

[2] F. Banhart, J. Kotakoski, and A. V. Krasheninnikov, ACS Nano 5, 26 (2011).

[3] J. H. Warner, E. R. Margine, M. Mukai, A. W. Robertson, F. Giustino, and A. I. Kirkland, Science 337, 209 (2012).

[4] O. V. Yazyev and S. G. Louie, Phys. Rev. B 81, 195420 (2010).

[5] J. Kosterlitz and D. Thouless, J. Phys. C 6, 1181 (1973); B. I. Halperin and D. R. Nelson, Phys. Rev. Lett. 41, 121 (1978); A. P. Young, Phys. Rev. B 19, 1855 (1979).

[6] C. Eisenmann, U. Gasser, P. Keim, G. Maret, and H.-H. von Grünberg, Phys. Rev. Lett. 95, 185502 (2005).

[7] K. Zahn, R. Lenke, and G. Maret, Phys. Rev. Lett. 82, 2721 (1999).

[8] U. Gasser, C. Eisenmann, G. Maret, and P. Keim, ChemPhysChem 11, 963 (2010).

[9] E. P. Bernard and W. Krauth, Phys. Rev. Lett. 107, 155704 (2011).

[10] V. Prasad, D. Semwogerere, and E. R. Weeks, J. Phys.: Condens. Matter 19, 113102 (2007).

[11] A. Pertsinidis and X. S. Ling, Phys. Rev. Lett. 87, 098303 (2001).

[12] A. Pertsinidis and X. S. Ling, New J. Phys. 7, 33 (2005).

[13] U. Gasser, E. R. Weeks, A. Schofield, P. N. Pusey, and D. A. Weitz, Science 292, 258 (2001).

[14] P. Schall, I. Cohern, D. A. Weitz, and F. Spaepen, Nature 440, 319 (2006).

[15] W. T. M. Irvine, M. J. Bowick, and P. M. Chaikin, Nature 11, 948 (2012).
[16] P. N. Ma, L. Pollet, M. Troyer, and F. C. Zhang, J. Low Temp. Phys. 152, 156 (2008).

[17] W. Lechner and C. Dellago, Soft Matter 5, 2752 (2009).

[18] W. Lechner and C. Dellago, Soft Matter 5, 646 (2009).

[19] J. P. Hirth and J. Lothe, Theory of Dislocations (Krieger, Malabar, FL, 1992).

[20] Y. Matsukawa and S. J. Zinkle, Science 318, 959 (2007).

[21] K. Arakawa, K. Ono, M. Isshiki, K. Mimura, M. Uchikoshi, and H. Mori, Science 318, 956 (2007).

[22] D. Hull and D. J. Bacon, Introduction to Dislocations (Butterworth-Heinemann, Oxford, 2001).

[23] K. Zahn, J. M. Méndez-Alcaraz, and G. Maret, Phys. Rev. Lett. 79, 175 (1997).

[24] F. Ebert, P. Dillman, G. Maret, and P. Keim, Rev. Sci. Instrum. 80, 083902 (2009).

[25] See Supplemental Material at http://link.aps.org/supplemental/ 10.1103/PhysRevE.88.060402 for additional technical details of the experiment and numerical methods.

[26] C. Dellago, M. M. Naor, and G. Hummer, Phys. Rev. Lett. 90, 105902 (2003).

[27] H. H. von Grünberg, P. Keim, and G. Maret, Soft Matter, Vol. 3 (Wiley-VCH Verlag, Weinheim, 2007).

[28] F. Nabarro, Theory of Dislocations (Clarendon, Oxford, 1967).

[29] H. H. von Grünberg, P. Keim, K. Zahn, and G. Maret, Phys. Rev. Lett. 93, 255703 (2004). 